\title{
A study of nowcast of Indian Monsoon rainfall over Mumbai using Rainymotion
}

Kunal Rustagi, Vipul Gote, Mahesh Mote, Jayesh Kothawade, Prof. Varsha Patil

\begin{abstract}
Affiliation: Dept of Electronics and Telecommunications, AISSMS IOIT
\end{abstract}

Twitter:@kunalr01, @vipul_gote_4

This manuscript is a preprint submitted to EarthArXiv. We openly share our work and to generate community discussion. Please feel free to contact the corresponding author for providing feedback or comments or further inquiries. We warmly welcome any suggestions.

Correspondence: kunal.rustagi@gmail.com 


\section{A study of nowcast of Indian Monsoon rainfall over Mumbai using Rainymotion}

\author{
Kunal Anil Rustagi \\ Dept. of ENTC \\ AISSMS IOIT \\ Pune, India \\ kunal.rustagi@gmail.com
}

\author{
Vipul Gote \\ Dept. of ENTC \\ AISSMS IOIT \\ Pune, India \\ vipulgote4@gmail.com
}

\author{
Mahesh Mote \\ Dept. of ENTC \\ AISSMS IOIT \\ Pune, India \\ mahesh.b.mote@gmail.com
}

\author{
Jayesh Kothawade \\ Dept. of ENTC \\ AISSMS IOIT \\ Pune, India \\ jayeshkothawade000@gmail.com
}

\author{
Prof. Varsha Patil \\ Asst. Professor, Dept. of ENTC \\ AISSMS IOIT \\ Pune, India \\ varsha.patil@aissmsioit.org
}

\begin{abstract}
We know rainfall is a very important factor in deciding the fate of a country. In an agrarian country like India where more than $70 \%$ of rural households are dependent on agriculture, agriculture contributes to nearly 17\% GDP of the country and is directly dependent on rainfall. Excessive rainfall can lead to floods, landslides, soil erosion and no rainfall can lead to drought-like conditions. Too much or too little rain can cause great havoc to the farmers. In order to prevent this damage to land and crops, rainfall prediction is the need of the hour. It can help us in future planning and preparing for the worst condition i.e. natural calamities like cloudbursts, floods, etc., thereby saving human life, vegetation, flora and fauna. Infrastructural losses worth billions can be avoided. In this paper, we tried to use one of the models provided in the rainymotion open-source library to predict rainfall in the nowcasting mode. The model is based on optical flow, a simple digital image processing technique, which tries to gauge the motion of the pixels, thereby creating a velocity field which is finally used to extrapolate the results. Using some verification methods, we have tried to predict the rainfall movement for over 60 to 80 minutes.
\end{abstract}

Index Terms-Rainfall prediction, Optical flow method, Rainfall nowcasting, Rainymotion

\section{INTRODUCTION}

Rainfall is amongst the most complex atmospheric processes and the most essential component in the water cycle. Water vapour formed due to air currents in the atmosphere condenses, which results in formation of rain clouds [1]. When clouds become heavy enough, ultimately it falls on the surface of earth due to the influence of gravity in the form of water droplets. Rainfall is the reason for fulfillment of almost every aspect on earth. But it is the intensity of rainfall which makes or breaks these aspects.

In India, around $75 \%$ of the total annual rainfall is from the South-west Monsoon winds during June to September which may reach India much before its due date or be delayed considerably. Though rainfall occurs from June to September but the actual rainy days are 40 to 45 days only. Heavy downpour in the Arabian Sea and the Bay of Bengal results in cyclones, floods, and excessive soil erosion. The lifestyle and economy depended largely on the amount and distribution of rainfall, as the whole country is predominantly agrarian. Rainfall from these winds is quite unreliable and highly variable. Also, distribution of rainfall is largely controlled by the relief features.

In 2019, India experienced the heaviest monsoon rainfall in 25 years. Instead of cheering up the agricultural heartland, the erratic monsoon left many crops damaged. Farming sector makes up around $15 \%$ of the economy and employs more than half of the country's people. The rains have replenished reservoirs and groundwater reserves, but destroyed the yields. The combination of prolonged dry spell followed by heavy rainfall increased pest infestation and disease, forced the farmer's expenditure on pesticides [2].

Flash floods, cloudbursts and heavy rains damaged the major crops- soybean, rice, cotton, sugarcane, pulses and vegetables. Destruction was not limited to crops, even buildings, roads, bridges were damaged and human lives were lost. Hence, the aim of this paper is to predict rainfall with good accuracy and minimize the devastation caused to life and property.

\section{DATA}

\section{A. S-Band Doppler Weather Radar}

The radar data used for the study had been obtained from the S-band Doppler Weather Radar (DWR) placed at the Navy Nagar in Colaba, Mumbai $\left(18.90^{\circ} \mathrm{N}, 72.80^{\circ} \mathrm{E}\right)$ and operated by the India Meteorological Department, Mumbai. The radar samples the clouds in 9 elevation angles, starting from $1^{\circ}$ $21^{\circ}$ in the Plan Position Indicator (PPI) scan mode with $1^{\circ}$ azimuth angle resolution and covering a radial distance of 240 $\mathrm{km}$ with a range resolution of $150 \mathrm{~m}$. The volume data of radar are obtained for every $10 \mathrm{~min}$. For the present analysis, the spatial variability of clouds as obtained from the PPI scan mode of the radar in $1^{\circ}$ elevation angle has been taken into 


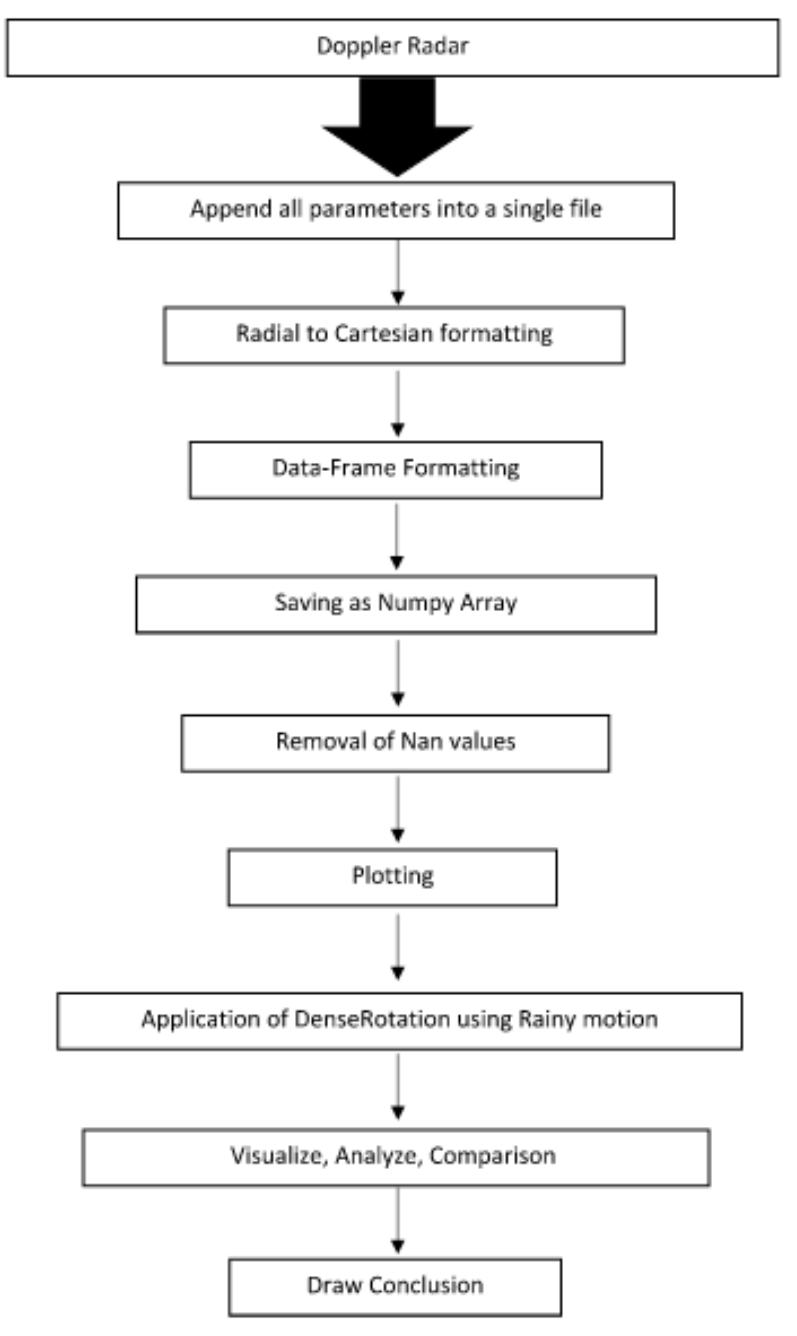

Fig. 1. Design Flow using Rainymotion

consideration. For the present study, data for July 09, 2018 has been considered.

\section{Methodology}

\section{A. Flowchart}

A basic overview of the various steps performed in this paper are depicted in the given flowchart Fig. 1.

\section{B. Procedure}

We begin by drawing data from the Doppler Radar situated in Mumbai. Rainfall events on 9th July 2018 for 3 hours each are under observation in this paper. The Doppler Weather Radar provides reflectivity values in network Common Data Form (netCDF) data at different elevation angles, but the project uses the first angle of elevation. The xarray [3] python library was used to read the data and important parameters were studied. A sample Plan Position Indicator (PPI) plot for one timestamp is shown in Fig. 2.

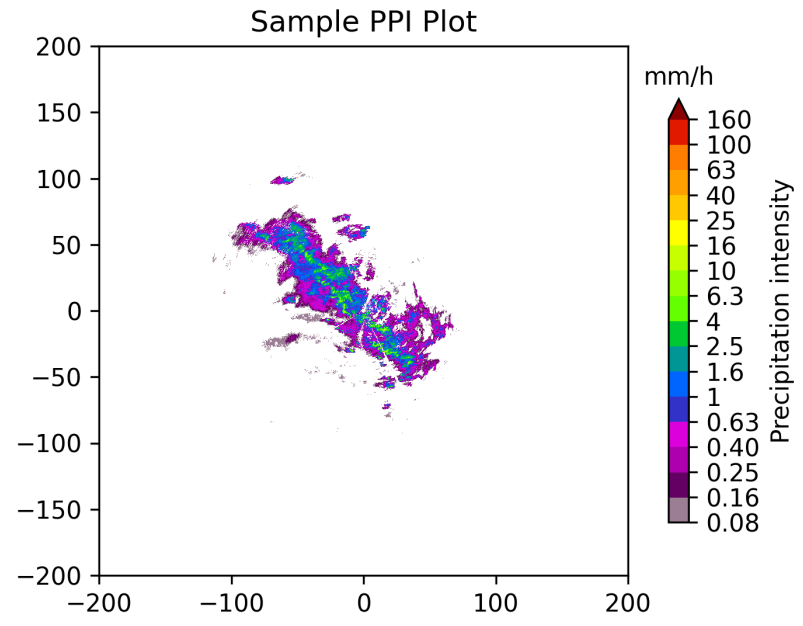

Fig. 2. Sample PPI Plot

Reflectivity data in the netCDF files was in polar form where bin dimensions were between 350 and 360, and radial dimensions were between 1570 and 1640, so reindexing of dimensions was carried out to [bin: 360, radial: 1600]. Following the study on clutter removal by Gabella [4], we decided to implement the same. A clutter-map was created for each image using the Gabella filter provided by the wradlib [5] python library and interpolated with the original images to remove the residual ground clutter. In order to perform image processing techniques like optical flow, creation of a continuous array of time separated radar images was necessary which was possible by converting polar data to Cartesian data.

After interpolation, we transform the reflectivity data into rain-rate ( $\mathrm{mm} / \mathrm{h}$ units) using Marshall - Palmer Law [6]. The data was split into train and test dataset having 3 consecutive images and 8 consecutive images, respectively. The last 2 images of the train dataset can be viewed in Fig. 3.

The rainymotion [7][8] library provides various models like Sparse, SparseSD, Dense and DenseRotation which are based on different optical flow algorithms. A Dense Inverse Search (DIS) [9] algorithm, which gives us an estimation of the
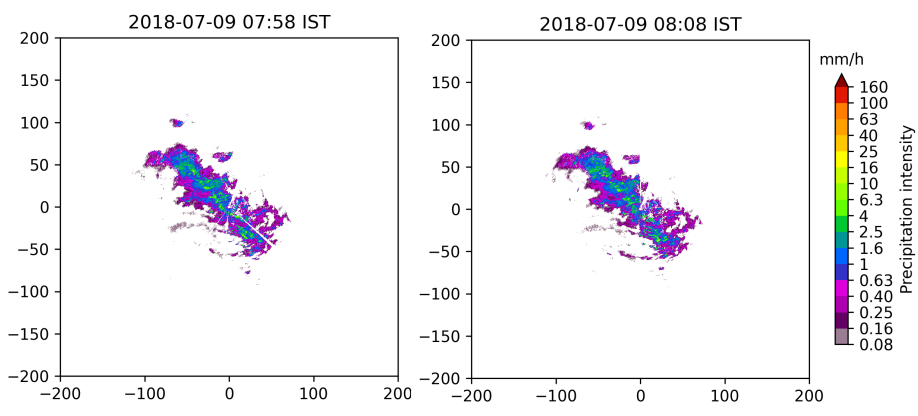

Fig. 3. Training set images 

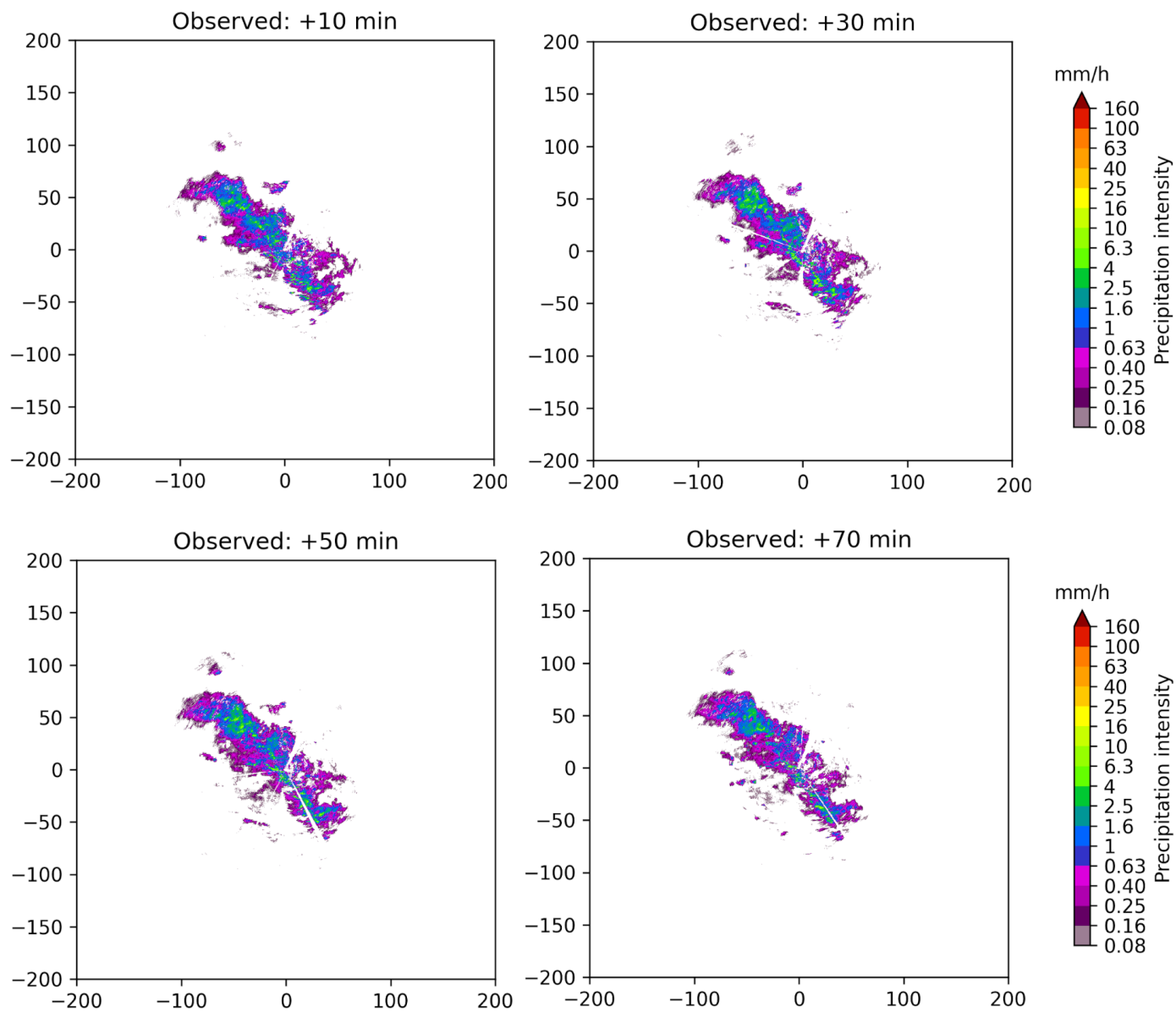

Fig. 4. Observed PPI Plots

velocity of each image pixel, is used by the Dense group of models. The model used in this paper is DenseRotation and the train dataset, where each image is separated by an interval of ten minutes, is used to obtain the velocity field. Based on the velocity field obtained, we extrapolated each pixel in a single step for each new lead time to derive future forecasts. This leads to the predictions where the original radar pixels appear to be shifted to a new location for each timestep. The size of the forecast array returned is equal to the size of the test array, which can now be verified using the metrics provided.

\section{RESUlts}

The presented technique was coded in Google Colab and VSCode IDE, and written in Python programming language. For the event in discussion, the DenseRotation model from rainymotion library was used to compute the nowcasts with lead times from 10min to $80 \mathrm{~min}$, where timestep is $10 \mathrm{~min}$. An example of the observed and predicted rainfall plots is depicted in Fig. 4 and Fig. 5 respectively for 10, 30, 50 and 70 lead times.

For verification, we used the Critical Success Index (CSI) score which is provided as a metric in the library and used a threshold value of 0.1 and $0.2 \mathrm{~mm} / \mathrm{h}$. Higher the CSI score, the better is the accuracy. The graphs for CSI score varying over time for both the thresholds is depicted in Figure 6.

\section{CONClusion}

Under the present scenario, the weather radar is a very important tool for nowcasting the severe rainfall event for any regions of the world. As such, the paper tried to portray by using the image and video processing technique the optical flow method for deriving the velocity field from consecutive images of weather radar. The study tried to analyse the performance of the DenseRotation model provided in the rainymotion library for nowcasting of rainfall events over Mumbai. In the end, we can conclude that we were successfully able to predict the rainfall over a period of 60 to 80 minutes which is indicated by the CSI graph. A CSI score of greater than 0.55 indicates a good forecast, which was observed in our results.

\section{FUtURE SCOPE}

There are several clear avenues for future work, let's start with the first approach to deal with the problem is using rainnet [10] model Architecture for the nowcasting of the various rain 

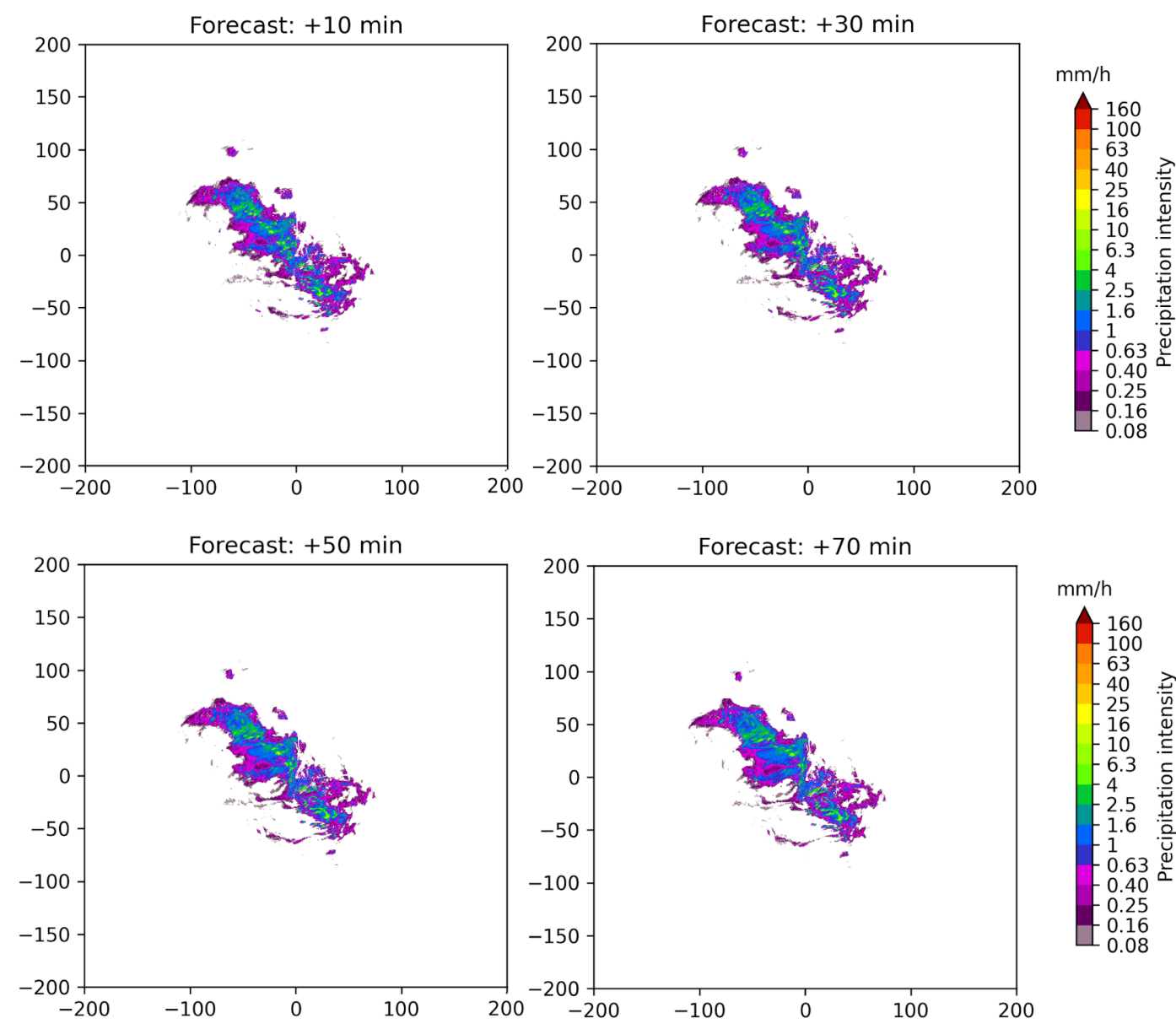

Fig. 5. Forecasted PPI Plots

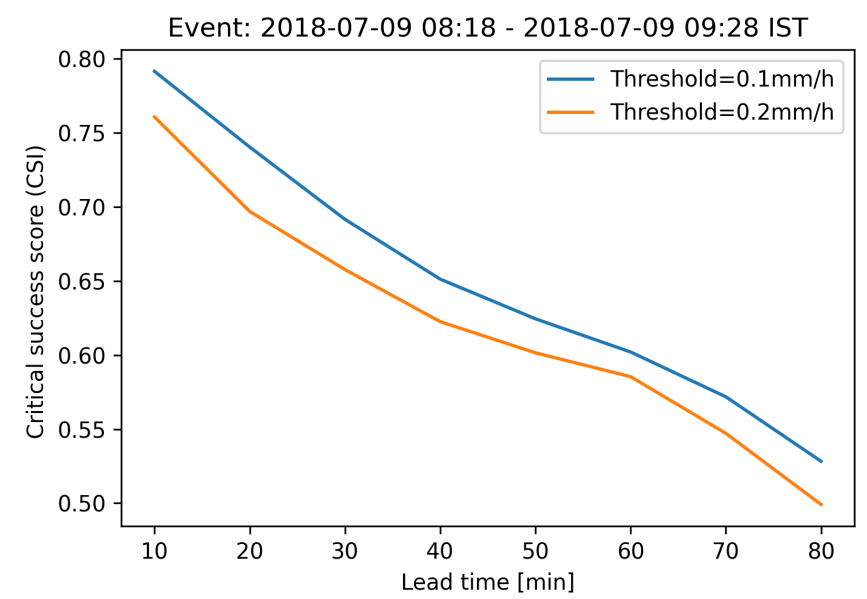

Fig. 6. CSI metric

events. This model architecture uses U-net architecture which has been giving very promising results in the forecasting of rain events.

Traditionally, we use Doppler radar for collecting the data but this data comes with a lot of drawbacks. For instance, there may be a huge structure like a mountain or a high rise building which would influence the readings. Also, there is a lot of static noise associated with Doppler radars. This can be eliminated by using satellite radars. This approach could be more efficient and less time consumable.

Finally, we can also opt for ensemble forecasting methods from different models and architectures

\section{ACKNOWLEDGMENT}

We would like to thank the Indian Meteorological Department for providing us the radar data. We also acknowledge the extensive support provided by the open source community at wradlib and rainymotion, especially Kai Mühlbauer and Georgy Ayzel for the many valuable discussions and educational help. We would also like to thank all those who guided us throughout this paper. 


\section{REFERENCES}

[1] Condensation and the Water Cycle in "https://www.usgs.gov/specialtopic/water-science-school/science/condensation-and-water-cycle?qtscience_center_objects=0\#qt-science_center_objects"

[2] Strong monsoon isn't always good news for farmers in "https://economictimes.indiatimes.com/news/economy/agriculture/strongmonsoon-isnt-always-good-news-for-farmers/is-heavy-rainfall-goodnews/slideshow/71538156.cms".

[3] Hoyer, S. and Hamman, J., 2017. xarray: N-D labeled Arrays and Datasets in Python. Journal of Open Research Software, 5(1), p.10. DOI: http://doi.org/10.5334/jors.148

[4] Gabella, Marco \& Notarpietro, Riccardo. (2002). Ground clutter characterization and elimination in mountainous terrain. Copernicus $\mathrm{GmbH}$. 305-311.

[5] Heistermann, M. \& Pfaff, Th \& Jacobi, Stephan. (2012). wradlib - An Open Source Library for Weather Radar Data Processing. 10511-.

[6] Marshall, J. S. \& Palmer, W. McK. J. Meteorol. 5, 165-166 (1948).

[7] Ayzel, G., Heistermann, M., and Winterrath, T.: Optical flow models as an open benchmark for radar-based precipitation nowcasting (rainymotion v0.1), Geosci. Model Dev., 12, 1387-1402, https://doi.org/10.5194/gmd-12-1387-2019, 2019.

[8] Ayzel, G., Heistermann, M., and Winterrath, T.: rainymotion: python library for radar-based precipitation nowcasting based on optical flow techniques, available at: https://github.com/hydrogo/rainymotion (last access: 28 March 2019), 2019.

[9] Kroeger, T., Timofte, R., Dai, D., and Van Gool, L.: Fast optical flow using dense inverse search, in: Computer Vision - ECCV 2016. ECCV 2016. Lecture Notes in Computer Science, edited by: Leibe, B., Matas, J., Sebe, N., and Welling, M., Springer, Cham, 9908, 471-488, https://doi.org/10.1007/978-3-319-46493-0_29, 2016.

[10] Ayzel, G., Scheffer, T., and Heistermann, M.: RainNet v1.0: a convolutional neural network for radar-based precipitation nowcasting, Geosci. Model Dev., 13, 2631-2644, https://doi.org/10.5194/gmd-13-2631-2020, 2020. 